\title{
WHAT CAN BE DONE FOR THE DEFORMED RHEUMATOID HAND?
}

\author{
O. J. Vaughan-Jackson, V.R.D., F.R.C.S. \\ Orthopadic Surgeon, The London Hospital, E.I
}

THE number of ways in which rheumatoid arthritis can affect a hand and the number of different deformities it can produce are so great that in seeking a coherent view of the problem we are faced with an immediate difficulty in sorting, tidying, and classifying all these factors. In a short article it is impossible to be exhaustive and one can only illustrate principles with examples. What follows illustrates a practical approach to the problem, doubtless full of gaps and perhaps with some downright mistakes in it-but it has served the author well in studying and working in what is a young subject, which we are only beginning to understand.

It must be recognized at the start that rheumatoid arthritis is unpredictable, except in its predilection for attacking women rather than men (in the ratio of $4: 1$ ). It attacks at all ages and in different guises. It may be infinitely slow or devastatingly rapid. It occurs in a 'wet' form with joint and tendon sheath effusions and exuberant synovial reactions, and in a 'dry' form in which bone and cartilage become eroded silently with little or no clinical involvement of soft tissues. It may progress inexorably, or quietly burn out at any stage. In the hand we recognize a type which hits first, mainly, and sometimes even exclusively, the wrist and inferior radio-ulnar joints. In another type the brunt falls on the metacarpophalangeal joints, in another on the proximal interphalangeal joints, in another on tendon sheaths. These are the main types though obviously overlapping occurs. But generally one hand tends to reproduce the pattern found in the other.

Not only, then, can we be faced with a rich variety of patterns of deformity but also the unpredictable timing of the onset of the disease in any joint or tendon ensures that any pair of rheumatoid hands will present us with young, old and intermediate lesions for consideration. It is important to realize that the treatment of these different stages tends to be along very different lines in each of three different joints in one hand.

The treatment of deformity in the rheumatoid hand demands an understanding not only of the actual mechanics of the deformity but also of the factors which influence it and change it through 5 out its progression from the mildest alteration in posture down to the complete dissolution and disorganization of a joint or tendon complex. The progression is as follows: a primary tendo of imbalance-due to various causes which will be. considered later-leads to a primary persistentक abnormal posture. If, as is usually the casef adaptive shortening or adhesions of soft tissues occur they convert the persistent abnormal postureg into a secondary fixed joint deformity. Progressives destruction of bone and cartilage, together with destruction of ligaments and joint capsules progressively changes this deformity into tertiary, modified, and final deformity, all too often. in fact a total disorganization of the joint.

It is very important not to have a static concept of deformity in this condition, and not to conside? only the ultimate dilapidations of the rheumatief hand. If we are to achieve anything our attentio must be fixed on the earliest changes-the firs\% hint of abnormal posture, or of those succession of abnormal postures that constitute abnormat patterns of movement. At that stage we may be able to prevent deformity. If we only consides fixed deformities we confine ourselves to salvage. procedures.

\section{Early Disease}

In early disease the hand, apart from being disabled by pain, adopts mildly abnormal posture? due to swelling of synovial layers. The tissues of joint capsule and associated tendons may b\& swollen or displaced. In certain situations evepp this slight swelling can upset tendon balances an produce a sequence of abnormal postures, that if to say an abnormal movement. For example, young man with fat, rather podgy hands had earl involvement of the metacarpophalangeal joints of index and middle fingers in each hand. The synovial swelling was of a degree only enough jus to obliterate the dimples on the dorsum of thes joints when he extended his fingers-the dimples remained over the unaffected joints. In eact affected finger the mechanism of pinch was altered. Normally to pinch index-tip to thumb-tie 
we stabilize the proximal phalanx in nearly full extension while we flex the two distal phalanges, finally flexing the metacarpophalangeal joints as we complete the pinch. In his case the affected fingers lay at rest in the abnormal posture of an early 'swan-neck' deformity-slightly flexed at metacarpophalangeal and terminal interphalangeal joints and slightly hyperextended at the proximal interphalangeal joints. When he tried to pinch, this abnormal posture was maintained and intensified. All he could achieve was flexion of the 'swan-neck' finger at the metacarpophalangeal joint, till at nearly $90^{\circ}$ flexion there was a snap, the displaced intrinsics flipped back into normal alignment, the proximal phalanx extended somewhat, and the terminal phalanges flexed into a normal pinch. A short while later he reported the development of yet another 'snapping swan-neck' in a ring finger which showed, on examination, no other change from its former condition than the disappearance of the dimple over the knuckle. So slight a degree of synovial swelling, then, can initiate the persistent abnormal posture which the progress of the disease is so exceedingly likely, by contractures and adaptive shortening of soft tissues, to ankylose into a fixed deformity.

Equally common in the early stage is the earliest hint of a boutonnière deformity-flexion of the proximal interphalangeal joint with extension of the terminal interphalangeal joint. Synovial swelling at the proximal interphalangeal joint distends and stretches the extensor expansion over the joint, producing slight flexion from relative lengthening of the central slip and slight extension of the terminal joint from tightening of the lateral bands over the swollen proximal joint. If untreated the lateral bands are slowly displaced downwards over the swelling till they lie in front of the axis of the joint and become persistent flexors of the proximal interphalangeal joint and extensors of the terminal interphalangeal joint and there is a fully developed boutonnière deformity (Fig. I).

Clearly if, as seems more and more certainly to be the case, rheumatoid involvement of synovial tissue shows no readiness to recur after synovectomy this procedure should give immediate correction of the deformity and lasting reliefand this in fact it does. There is moreover some urgency about it since, especially at the proximal interphalangeal joint, the active rheumatoid synovium exhibits an almost malignant local destructive capacity. Not only does it soon erode bone but it distends the extensor expansion and by producing multiple herniations through it so destroys its integrity that it is virtually impossible to repair and replace its delicate mechanism. Early exploration and synovectomy in these joints is an urgent necessity.

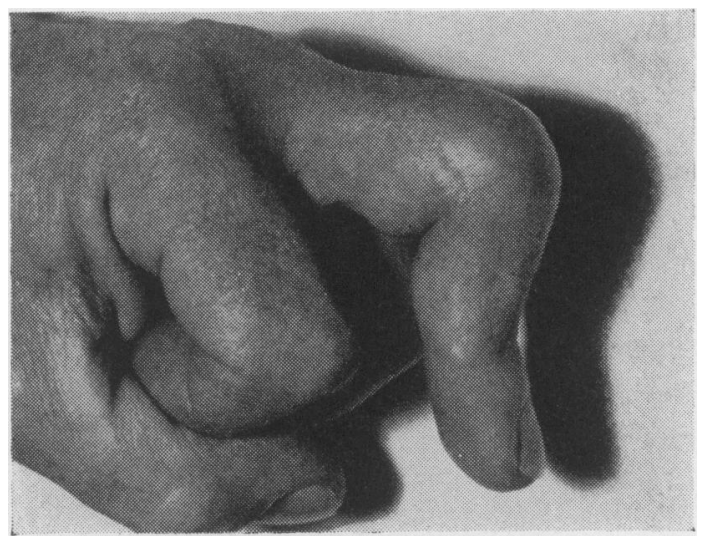

Fig. 1.-Typical boutonnière deformity.

\section{Intermediate Disease}

Passing from the early stage the patient, and her hands, enter upon a relatively long intermediate stage in which the disability may not be very great. No great destruction of joints or tendons has occurred. Everything still moves-albeit rather creakily and with variable discomfort. The odd boutonnière deformity appears and is quietly compensated for. Snapping swan-necks, trigger fingers, ulnar drifts all slowly appear. Occasionally tendons rupture, perhaps through attrition of the extensors over the lower end of the ulna causing fingers to drop at the metacarpophalangeal joints, or perhaps through degenerative changes, producing dropped thumbs. A swan-neck appears, due perhaps to a ruptured sublimis giving the extensor mechanism an unopposed preponderance at the proximal interphalangeal joint.

This is the phase of slowly progressive soft tissue changes in which bony damage is present but not yet to a degree to cause real disorganization of joints. It is marked by an increasing display of fortitude and adaptation by the patients, a majority of whom, except in their periods of greater discomfort, develop a wry form of humour about their troubles. Their fortitude commands respect but that they should be forced to display it is a considerable disgrace to us. For far too long have we used it as something to shield us from the necessity of looking at our patients, of analysing what we see, of working out what has happened and devising means of repairing it and preventing its extension or recurrence. Now that we are, increasingly many of us, really looking at these cases we realize with dismay how blind we have been and what opportunities we have missed in the past.

From what has been said it is clear that this intermediate phase calls in general for soft tissue procedures. With ruptured tendons we have to 


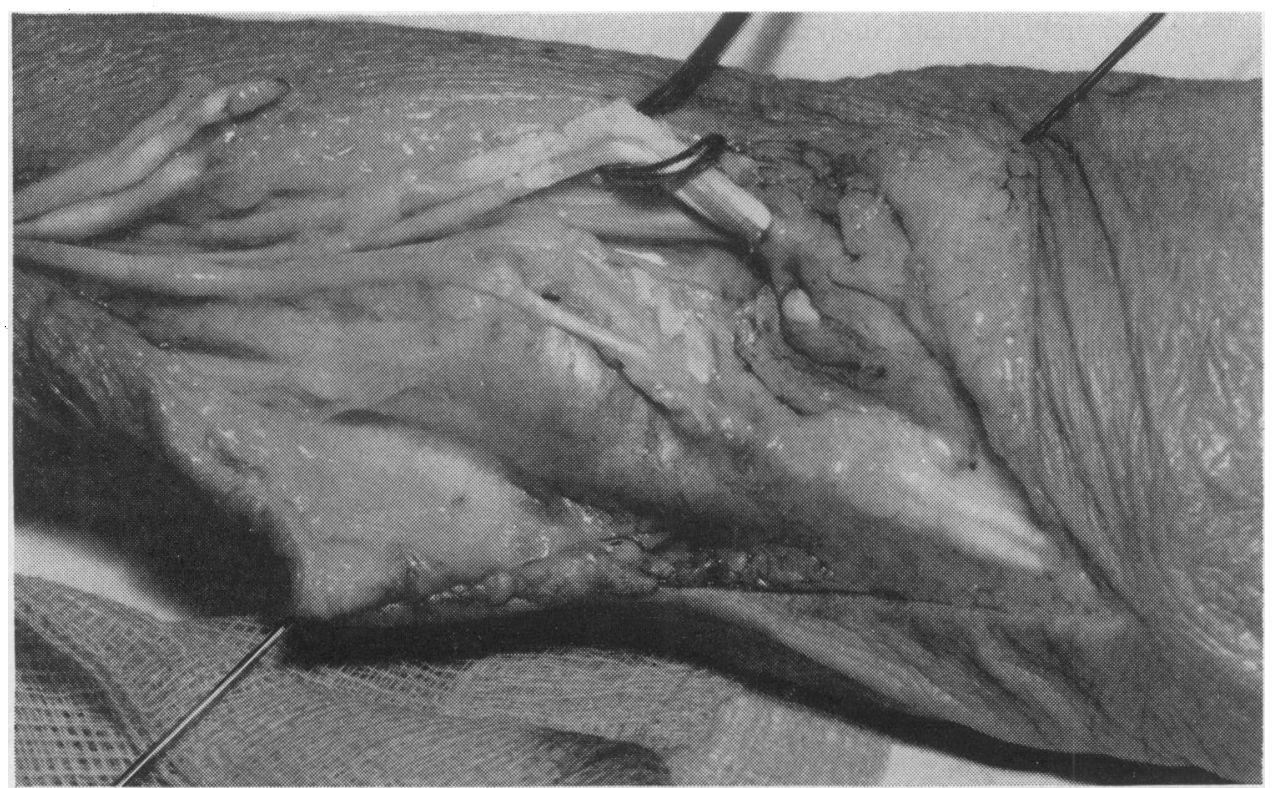

FIG. 2.- Typical rupture of extensor tendons by attrition on a roughened lower end of ulna. The black spot near the centre is the hole in the floor of the tendon compartment through which the ulna 'attacked' the tendons. Ruptured tendon ends lie to left and right and partly abraded tendons lie in the grip of the hook.

remove the cause and repair the tendon. Removal of an eroded lower end of ulna is an essential prerequisite of a repair, by any technique, of an attrition rupture of the extensors, otherwise the repair will be abraded in turn. It is easily carried out through the same dorsal incision that exposes the ruptured tendons and seems to be an operation without penalties or risks. Moreover it carries with it a bonus in that it relieves the usually painful state of the affected inferior radio-ulnar joint and also relieves the restriction of pronation and supination that results from involvement of this joint (Fig. 2).

Fingers stiff, or stiffening, in extension may be due to ruptures, attrition or otherwise, of flexor profundus or sublimis in the carpal tunnel. A dropped thumb may result from rupture of extensor pollicis longus.

There is nothing peculiar or unusual about the techniques of repair of these tendons-the pitfall is to fail to diagnose the ruptures and to ascribe thoughtlessly the persistent posture of, say, flexion at the metacarpophalangeal joints, to arthritis, to joint changes, when the cause lies in tendons ruptured remotely, at the wrist. It is not often possible to carry out end-to-end repairs. Free tendon grafts can work very well but whenever possible it is better to transfer a living tendonsuch as extensor indicis to repair a ruptured extensor pollicis longus. There is then only one suture line to go wrong and the transferred tendon has a blood supply. The prospects are clegr better than for two suture lines, one at either enj of a length of dead tendon. Often, too, witi tendons of similar function and a common musce belly, it is possible to attach a distal tendon stum to an intact neighbour (such as flexor profundrs to flexor profundus), and regain excellent function.

Short of actual ruptures tendons can be han: pered by synovial swellings and prevented from making their normal contribution to tendoñ balances. The trigger finger is an obvious example easily cured by excision of tendon nodules or by synovial clearance of the affected tendon sheaths: Diffuse involvement of the sheaths may hampes say, a flexor sublimis tendon so much as to give the extensors a preponderance at the proxima interphalangeal joints, so producing a swan-neck deformity.

Tendons can be displaced and their action thereby altered. Distension and invasion of the extensor expansion by diseased synovium at th metacarpophalangeal joints can produce an ulnăb deviation of the fingers in two ways. First the common extensor tendon can be, as it were 'shouldered' off the metacarpal head by the synovial swelling, assisted, it is thought, by two factors-first a tendency, progressively great 8 from index to little finger, for the digit to extenid in any case in some ulnar deviation, and second by gravity acting on the fingers with the hand in the mid-pronation-supination position in which $\frac{O}{8}$ 


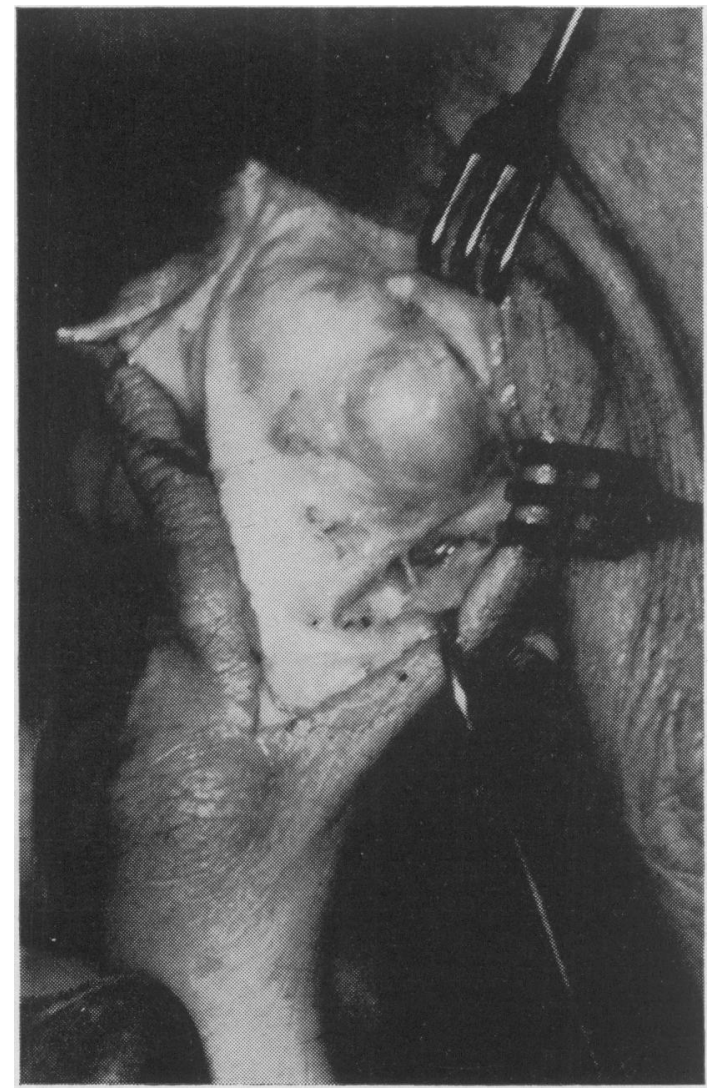

Fig. 3.-A metacarpophalangeal joint exposed from the dorsum. An obvious synovial swelling bulging towards us has shouldered the common extensor tendon off the metacarpal head to the left. It has pushed the lateral band of the expansion downwards to the right (opposite the middle retractor). Between them the taut transverse fibres are clearly obstructed in their proximal travel by the swelling.

is held for most of the time. Second, a synovial swelling can project proximal to the transverse basal fibres of the expansion on the radial side between the common extensor and the interosseous tendon, displacing the latter forwards in front of the transverse axis of the metacarpophalangeal joint. The interossei then become persistent flexors of the metacarpophalangeal joint and the proximal travel of the radial half of the expansion is obstructed. The ulnar half being unopposed, there is an immediate ulnar preponderance and ulnar deviation is produced (Fig. 3).

Removal of such a synovial mass early before irreversible distortion occurs can and will cure the deviation. In other cases the deviation is less easy to understand in the absence of such mechanical obstruction but a pleating operation on the radial side of the metacarpophalangeal joint can

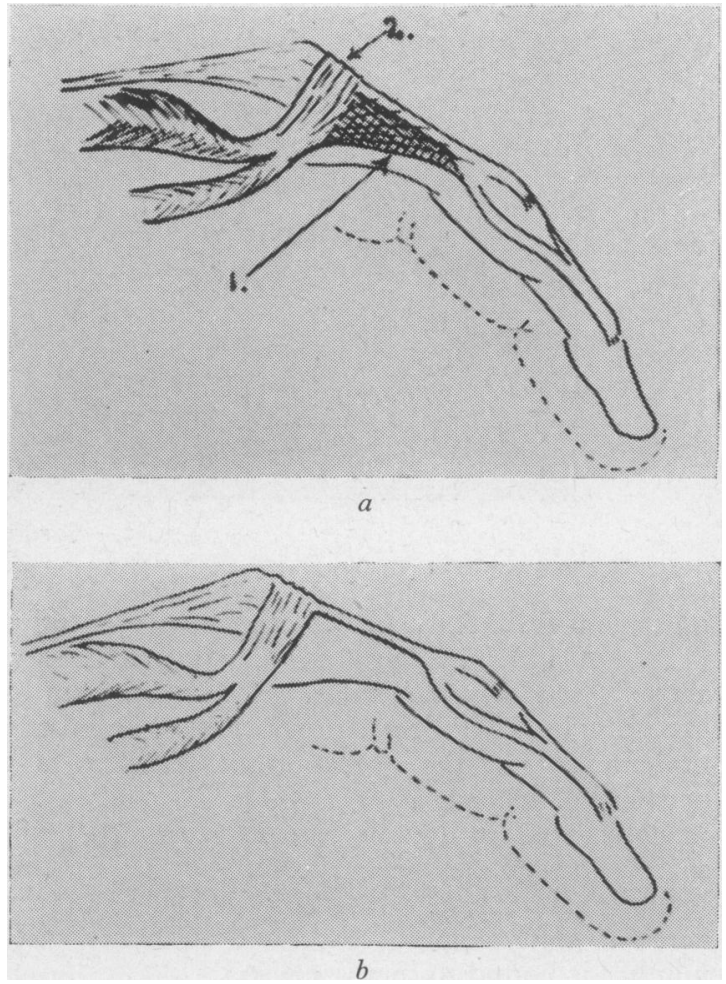

Fig. 4.-(a) Diagram of extensor expansion. The hatched triangular area is removed in the Littler release operation. (b) With this portion of the expansion removed the common extensor tendon is left intact, together with the transverse basal fibres of the expansion.

replace the extensor tendon on the metacarpal head. It is important to maintain this correction with a plaster, or some form of splinting, preferably of the active variety, while the capsulorrhaphy heals and consolidates.

Distension and stretching of the volar aspect of the capsule and of the collateral ligaments of the metacarpophalangeal joints may allow forward subluxation of the bases of the proximal phalanges. This at once disturbs the delicate balance of the phalanges-commonly producing a swan-neck deformity. Often this is only amenable to a later salvage procedure-a form of arthroplasty, but one simple device may save the day and is worth trying. A simple leather wristlet has a hook on the dorsum from which four elastic loops are passed over the fingers and the tension adjusted till they lie along the dorsum of the hand, pulling in a backward direction on the base of the proximal phalanges. This counters the forward subluxation and has in some cases abolished the tendency for a swan-neck to develop.

In the fully developed swan-neck deformity resulting from tight intrinsic muscles the release 


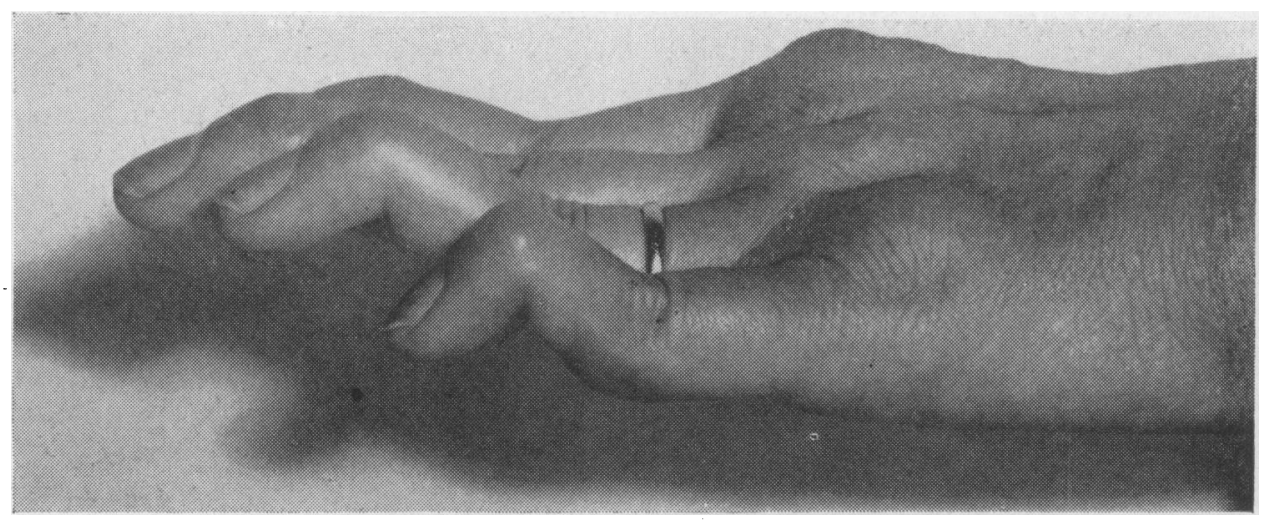

Fig. 5.-Severe swan-neck deformity.

operation devised by Littler can be very effective (Figs. $4 a$ and $b$ ). The deformity does, however, show a tendency to recur. Its mechanism is exceedingly complicated and still imperfectly understood but the operation nevertheless is a very useful procedure.

Thus there are already a considerable number of soft tissue procedures in common use in this intermediate phase. As our knowledge of the detailed mechanism of the hand increases their number is bound to increase also.

\section{Late Disease}

Coming now to the ultimate dilapidations our operative procedures tend to be much more upon bone and joint and less upon soft tissues. A row of dislocated metacarpophalangeal joints in full ulnar deviation and with gross bone damage is not amenable to soft tissue procedures. The metacarpophalangeal joint in the finger is the one joint in the hand that should seldom if ever be arthrodesed (though this is not true of the thumb). Accordingly attention has been focused on arthroplasty. The competing claims of stability and mobility have produced some widely different solutions. Arthroplasty of these joints by simple excision produces a flail condition of the fingers which is at first alarming. However, it can release tight intrinsics and abolish swan-neck deformities and it is surprising how often the shape of objects, that are gripped in common everyday use, seems to guide the flail fingers into parallel alignment so that the grip is effective.

In any case almost anything is likely to be more effective than fingers freezing into the maximum swan-neck deformity where function is minimal (Fig. 5). In such cases reversal of the fixed hyperextended position of the proximal interphalangeal joints by serial manipulations, and plasters, assisted in the more fixed deformities by capsulotomies of these joints, can transform these hands, provided only that there is a useful ranige of movement at the metacarpophalangeal joints. If fingers are doomed to be stiff let us at least take pains to ensure that they are stiff in flexion and not in hyperextension.

Returning to the metacarpophalangeal joints an attempt to diminish the instability of an arthroplasty by excision was made by Fowler who shaped the head of the metacarpal into a transverse wedge the edge of which was in contact with $t$ base of the proximal phalanx. The latter çup then flex and extend by rocking on the edge of his wedge. This can be an excellent arthroplasty owing to adhesions occurring between the bare bone of the wedged metacarpal head and the surrounding soft tissues the degree of mobilin that can be obtained is rather unpredictable. The metal hinges pegged into the medullary canals of phalanx and metacarpal introduced by Brannom and modified by Flatt have the advantages of not adhering in this way, of maintaining length and with it the normal tensions in the related tendon, and of conferring satisfactory lateral stability on these joints. Abduction and adduction at the metacarpophalangeal joints are, of course, sacrificéd (Fig. 6).

While chastening experiences with metallic and other implants in other parts of the body, notabły the hip, oblige us to be wary of such prostheses, the indications are all so far favourable. It quite certain that these devices are capable of restoring nearly normal function. How long they will last, and how long they will remain in position in bone softened by the rheumatoid process remaings to be seen. But at least we do not, most of us, walk on our hands so that the prostheses are spared the disrupting forces that have beset some others. Watchful optimism seems to be the appropriate attitude.

At the wrist, on the other hand, an arthrodes is often highly successful. It is quite unrealist. 


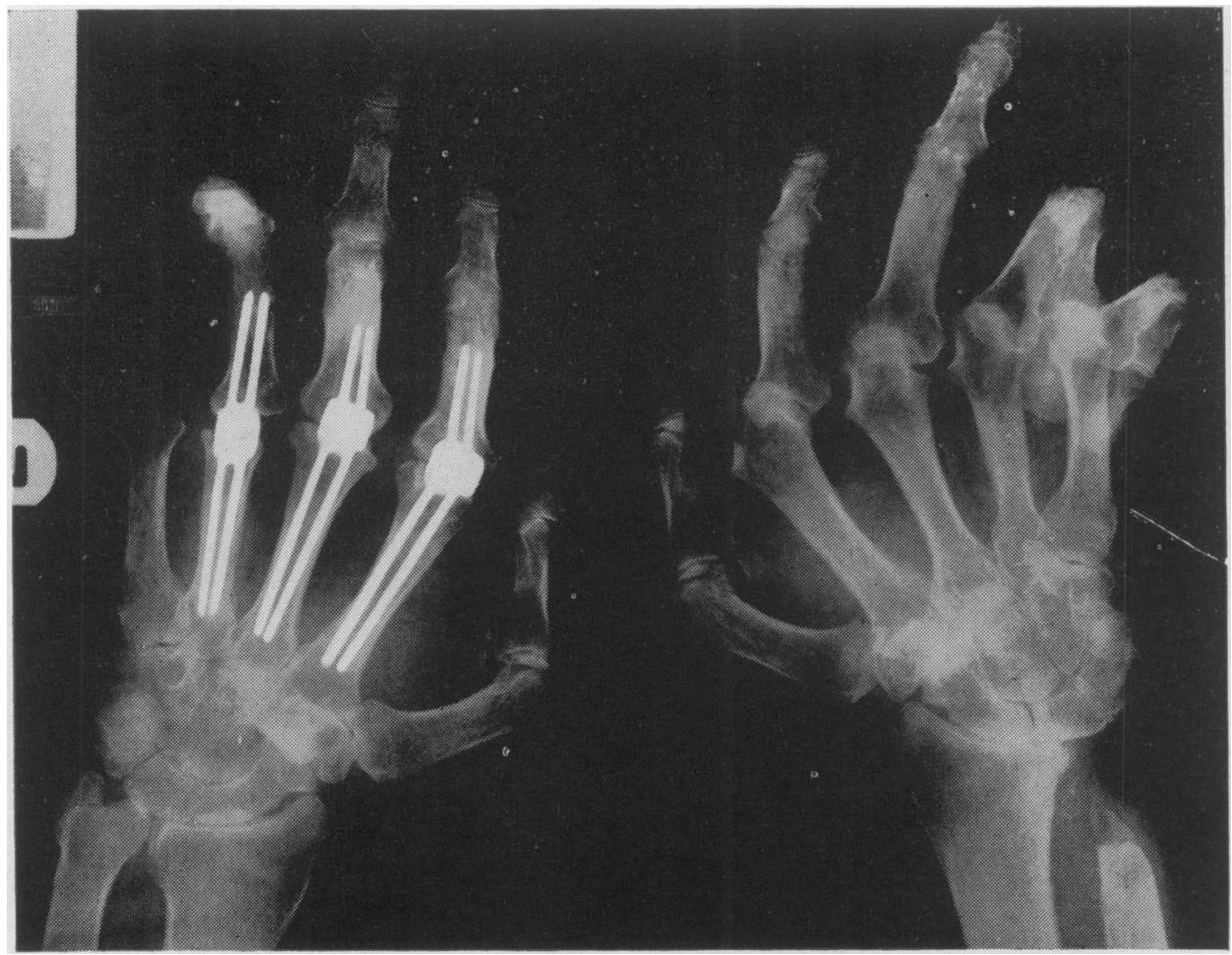

FIG. 6.-Flatt hinge prostheses in situ in metacarpophalangeal joints. Before operation these joints were in a similar state of damage and subluxation to that shown in the opposite hand.

to attempt to control a severely damaged and painful wrist by external splints. All that can result is the imperfect control of pain and a totally disproportionate decrease in function (this must be carefully distinguished from splinting for an acute flare in a relatively undamaged joint, which is quite a different problem).

The wrist is an easy joint to arthrodese by any of several well-tried methods and in these cases arthrodesis is a certain 'winner'. If the procedure includes an excision of the lower end of the ulna, pronation and supination are restored. The ensuing stability and relief of pain can in addition transform quivering, stammering, ineffective fingers into effective units again, despite their being themselves damaged, so great is the effect on the hand as a whole of this master joint - the wrist. Provided only that elbow and shoulder are not so damaged that the necessary compensatory movements are impossible, an arthrodesis of the wrist is one of the most reliable shots in the hand surgeon's locker (Fig. 7).

There are other procedures, of course, for which there cannot be room in a short survey. The aim of the surgeon dealing with the rheumatoid hand is not, however, to be prolific in giving birth to new procedures, however necessary these may be nevertheless. It is, instead, to intervene early enough to prevent damage and to restore disturbed balances and patterns of movement by procedures that can be simple and minor because they are timely.

\section{The Place of Synovectomy}

Early synovectomies have already been referred to. Not too much emphasis has been placed upon them, however, as a survey of late results is not yet possible. Provided, however, it is understood exactly what can be claimed for the method its continued use may properly be advocated.

The former poor reputation of synovectomy derived from the fact that it was always employed late in severely damaged joints. However, when one considers that damage to bone, articular cartilage, ligament and tendon in rheumatoid arthritis only occurs where the diseased synovial tissue is in contact with these structures the early removal of the synovium is an obvious method to try. The results have been uniformly encouraging but the follow-up is only about two years in the really early synovectomies, undertaken before any bony damage can be seen in the radiograph. In close to 100 cases no proved recurrence has yet been seen. This, of course, seems too good to be 


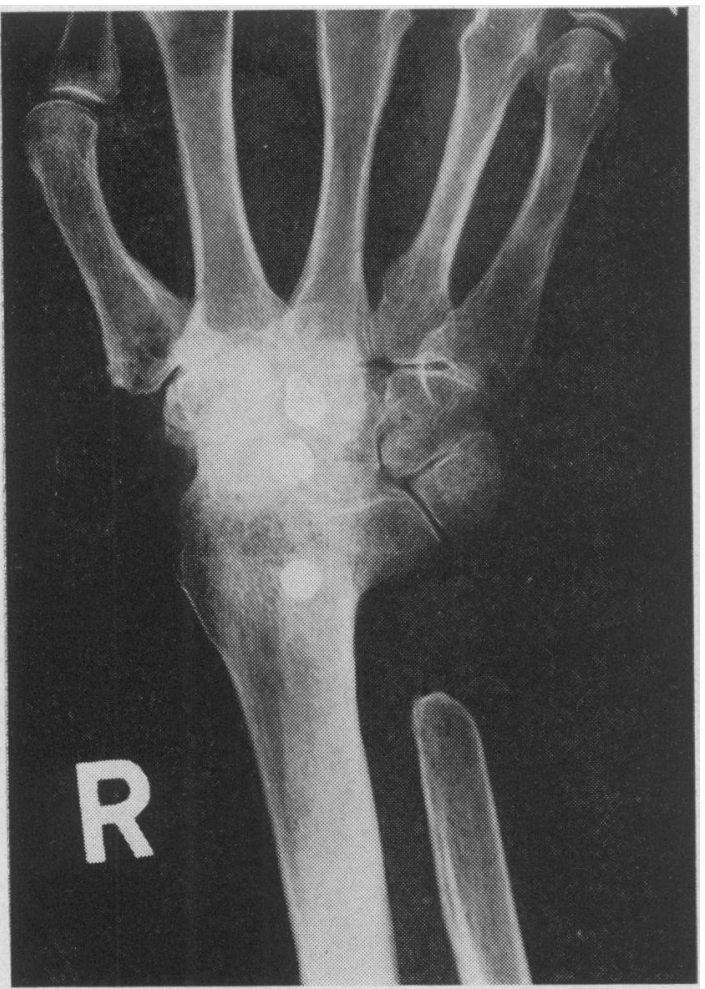

FIG. 7.--Radiograph of wrist arthrodesed using a dorsal iliac bone graft. If the graft can be securely slotted, one end into the bases of the second and third metacarpals, and the other into the lower end of the radius, the securing screws may be dispensed with.

true and recurrences must surely occur sooner or later. Moreover, it must be realized that unequivocally involved synovium need not necessarily produce a clinically evident swelling. The dis- appearance of clinical synovial swelling undes steroid therapy is manifestly not to be regarded as the equivalent of a synovectomy. Equally the absence of clinically detectable swelling aftew synovectomy is not to be interpreted as proof of non-recurrence. We have to wait patiently foi opportunities of second explorations and biopsies of these joints. We must continue then to scrutinize serial radiographs for the appearance ox extension of bony damage.

Meanwhile we can take comfort from the following facts. The joints of the wrist and hand stand the surgical trauma of really early synovectomy very well indeed. The patients are delighted with the relief of pain. Functional recovery complete and rapid. In no really early case has any joint come to harm as the result of synovectom In later cases where bone erosion exists tendo and capsule tend to adhere to the raw bone laif bare by synovectomy and the functional results are in inverse proportion to the degree of bonos damage.

In addition to the hope of local cure and the certainty of local relief from pain must be adde the importance of synovectomy in removing locat obstructions and restoring upset tendon balances already referred to.

In tendon sheaths the synovectomy is a dess urgent matter as tendons show much greagrt resistance to invasion than bone and articular cartilage. But sooner or later they succumb if in synovectomy is performed. The removal of large obstructing masses of affected synovium can hame an almost magical effect in releasing the stof rheumatoid hand, relieving pain and the paræ thesiæ of compressed median and ulnar nerves, and restoring those tendon balances whose importance in the maintenance of normal postures and movements cannot be over-emphasized.

\section{REFERENCES}

BÄCKDAHL, M. J., and MYRIN, S. O. (196r): Ulnar Deviation of the Fingers in Rheumatoid Arthritis and Its Surgich Correction, Acta chir. scand., 122, 158.

Brewerton, D. A. (1957): Hand Deformities in Rheumatoid Disease, Ann. rheum. Dis., 16, 183.

Carroll, R. E., and TABer, T. H. (1954): Digital Arthroplasty of the Proximal Interphalangeal Joint, $\mathcal{F}$. Bone $\mathcal{F} t$ Surg. 36A, 912.

Flatt, A. E. (1963): 'The Care of the Rheumatoid Hand'. St. Louis: C. V. Mosby. (1960): The Prosthetic Replacement of Rheumatoid Finger Joints, Rheumatism, r6, 90.

- (1962): Surgical Rehabilitation of the Rheumatoid Hand, Ann. roy. Coll. Surg. Engl., 31, 283.

Pulkki, T. (r96i): Rheumatoid Deformities of the Hand, Acta rheum. scand., 7, 85.

Riordan, D. C., and Fowler, S. B. (1958): Surgical Treatment of Rheumatoid Deformities of the Hand, $\mathcal{F}$. Bone $\mathcal{H}_{t}$ Surg., 40A, I 431 .

Sperling, I. L. (1950): Rheumatoid Polytendovaginitis, Ann. rheum. Dis., 9, 43.

Straub, L. E., and Wilson, E. H. (1956): Spontaneous Rupture of Extensor Tendons in the Hand Associated with Rheumatoid Arthritis, $\mathcal{F}$. Bone $\mathscr{f} t$ Surg., 38A, 1208.

Varnio, K. (1957): Carpal Canal Syndrome Caused by Tenosynovitis, Acta rheum. scand., 4, 22.

- (1959): The Role of Surgery in the Rehabilitation of Rheumatoid Arthritis Patients, Proc. 4th European Rheume $t$. Cong., p. 223.

Vaughan-Jackson, O. J. (1959): Attrition Ruptures of Tendons as a Factor in the Production of Deformities in the Rheumatoid Hand, Proc. roy. Soc. Med., 52, 132.

- (1962): Rheumatoid Hand Deformities Considered in the Light of Tendon Imbalance-I., $\mathcal{F}$. Bone $\mathfrak{F} t$ Surge, 44B, 764 . 